\title{
Approach of immersive adaptive learning for Virtual Reality simulator
}

\author{
Alieski Véliz Vega', Omar Correa Madrigal², Vlada Kugurakova ${ }^{3}$ \\ ${ }^{1}$ Visialization and Virtual Reality Research Group - Universidad de las Ciencias \\ Informáticas (UCI), La Habana - Cuba \\ ${ }^{2}$ Visualization and Virtual Reality Research Group - Universidad de las Ciencias \\ Informáticas (UCI), La Habana - Cuba \\ ${ }^{3}$ Higher School ITIS - Kazan Federal University (KFU) - Kazan, Russian Federation \\ veliz@uci.cu, ocorrea@uci.cu, vlada.kugurakova@gmail.com
}

\begin{abstract}
The need to improve teaching-learning processes promotes the development of Virtual Reality applications with adaptive learning approaches. Adaptive learning considers the premise that each person learns differently, at their own pace, considering the characteristics of the student. This paper describes the main components of an immersive adaptive learning approach for Virtual Reality simulators.
\end{abstract}

\section{Introduction}

Technological advances introduce dynamism to the way content is displayed and consumed. The introduction of novel technology benefit fields such as medicine, education, and industry. The use of virtual learning environments has become a complement to face classes at different levels of education, however, it would be better used if they constituted immersive environments. Numerous studies show that to achieve greater motivation in students it is necessary an immersion's degree of student. In this sense, the application of Virtual Reality (VR) plays a fundamental role. This takes place in a safe environment that allows reconstructing the surrounding reality and control the different stimuli to which an individual is subjected. Immersive environments provide multi-sensory, playful, multimedia, and interactive stimulation, and allow to control stimulation conditions and repeat actions as in the real world.

Researches carried out in the context of immersive 3D environments show a strong connection between virtual education and immersive environments as a current trend (Comas-González et al., 2017). VR-based training is particularly effective in the education field in a wide variety of subjects, reflecting the results in medicine, biotechnology, the military industry, sport, among other fields. Studies on adaptive learning systems are conducted with the premise that each person learns differently. This study presents an approach of immersive adaptive learning for VR simulators.

\section{Adaptive learning system}

Adaptive learning is "a learning model based on technology that can detect the students individual situation, context, learning needs and style, and the state of their learning process dynamically, and act according to them" (Real-Fernández, Molina-Carmona, 
Pertegal-Felices, \& Llorens-Largo, 2019). Other researchers have identified four categories of adaptive learning environments: adaptive interaction, adaptive course delivery, content discovery and assembly, and adaptive collaboration support (Paramythis \& Loidl-Reisinger, 2004)(Kara \& Sevim, 2020). Adaptive learning systems are concentrated on various fields of academic research such as automated learning, memory and cognitive load theories, intelligent tutoring systems, adaptive hypermedia systems, among others (Weber, 2012)(Chen \& Zhang, 2008). Among the most influential categories are content-driven systems, which are based on interactions and metadata generated by user interaction with content, as well as performance monitoring. In this category, we can highlight the example of Cheryl Lemke, and it's model called "adaptive intelligent learning"(Lemke, 2013). This model is also defined as digital learning in which the student's actions are captured and used to guide their own learning experiences, by adjusting their rhythms and plotting an independent route for it.

In an adaptive learning system, feedback must occur immediately so that students stay more focused and can easily self-correct. Continuously adaptive systems can provide personalized feedback to both multiple-choice and free-answer questions instantly or almost instantly. The result is a pace that leads to mistakes reduction, experimentation, iterative development, and quick learning. Adaptive learning allows each individual's learning to be personalized using resources offered from a platform, covering the content in-depth in a rational way and constituting a tool for both the student and the teacher. The virtual learning platforms that incorporate adaptive learning in their conception, are based on data collection techniques to learn from each user and provide an adaptation to the content that shows them. The processing of the information collected from each user allows the platform to keep track of the progress of each student and provides the teacher with this information to track each student more exhaustively and individually.

After the study of various platforms (among these Alexia, Smart Sparrow, NEO LMS, LearnSamart, Knewton), it is considered that among the most relevant aspects to be taken into account in an adaptive learning system are instant feedback, collection and processing of data on the student progress, and adjustment of content to correspond with the student progress. The above aspects are essential characteristics to be considered also in the implementation of self-adaptive immersive learning systems.

\section{Immersive Virtual Reality Environments}

Virtual Reality (VR) environments are classified within two main categories: nonimmersive (projection screens, desktop computers) and immersive (Head Mounted Displays, HMD). Adaptive learning platforms are not VR environments; however, its main contribution is the ability to adjust content and measure the progress of each student, ensuring that each student maintains its own learning pace adjusted to their abilities. Despite the advantages and their significant contributions in the educational context, students do not experience immersive learning, since the consumption of content traditionally takes place in a flat perspective.

Immersive learning is the process of learning with the usage of a simulated environment that allows students to connect as real experiences within a safe environment. Through these experiences, motivation, and commitment to the activity being undertaken increases and leaves an impression on the student's psyche that favors the memorization 
of the content. In a non-immersive virtual environment, the user has the sensation of seeing everything from a window. However, using HMD, the user experiences being inside the virtual environment, although not physically, but mentally.

The low cost of mobile applications with virtual reality has allowed the development of very innovative applications with this technology in sectors such as education, entertainment, tourism, medicine, and sports. Especially for educational purposes, immersive VR generates a series of experiences promoting learning more innovatively. In this sense, some studies address the satisfaction of students from the experience of learning through immersive virtual platforms, highlighting (Comas-González et al., 2017; Pfandler, Lazarovici, Stefan, Wucherer, \& Weigl, 2017):

- The acquisition of new skills and the satisfaction of applying them effectively.

- Motivation to learn and show better performance.

- The acquisition of knowledge from the exploration of a non-real interactive environment.

- Sensory stimulation and sense of presence in that environment.

VR applied in different contexts has reached an important place among current technologies. Virtual environments create a safe environment without risks and allow skills to be developed through repetition. A common problem with VR systems for training is that users experience the same training routines, as these VR systems are not customized for individual learning patterns (Vaughan, Gabrys, \& Dubey, 2016). A completely immersive and self-adaptive virtual learning environment must be able to adjust the contents and measure the advancement of each individual and adjust to their physical abilities and at the same time, provide quick feedback to make the most of all the variables involved in learning.

\subsection{Virtual Reality selft-adaptive technology}

VR simulators can be adapted to the capacities of the user. Adaptation occurs through haptic devices and other accessories that allow movement and other functions. Another type of adaptation takes place through the software with content and user interface adjustment, supported by the use of stereoscopic devices and hand controllers. Basic elements of VR-based training simulation include haptic devices, autonomous agents, adaptive technologies, feedback evaluation and classification, and HMD. A study realized in this field point to the evolution of self-adaptive technologies in VR training (Vaughan et al., 2016). With the evolution of adaptive technologies in the field of VR, the areas of application of VR training systems are increasing also, highlighting: the area of medicine, education, commerce, and the military industry. The self-adaptation of VR systems, as well as adaptive learning platforms, requires the processing of users' data records. Among the most common success in the field of self-adaptive virtual simulators are those designed to train pilots, drive vehicles, do e-commerce, and military training (Vaughan et al., 2016)(Lele, 2013). A VR environment with an adaptive learning approach makes decisions about the next and best step to take during user training. Different methods to perform this behavior focus on the storage and processing of user data during training. Data provides a record of results, scores, errors 
made and other information about the user and the activities he has performed. This process involves techniques that support decisions about the next and best step to take.

Other studies conduct a discussion on how to adapt a Virtual Learning Environment (VLE), from different components of a VLE: scene and objects, behavior, user interaction; communication, and sound (De Troyer, Kleinermann, \& Ewais, 2010). In this approach, the adaptation from one or more of these components is pointed out. Concerning one type of adaptation of objects, the adaptation of object display (the way to show or hide an object) is pointed out. Other parameters are also defined for the adaptation of other components. Similar to the process of adaptation of a Virtual Learning Environment, they alter the approaches to a VR system. To adapt content to the learner, it is also necessary to have information about the learner (personal preferences, background knowledge, etc.) and to keep track of learning progress at runtime. This is usually carried through the use of a user-profile, a user model, or learner model.

Authors as (Wojtok, Bab, \& Hirsch Martin, 2018) introduce an approach of adaptive learning in VR composed in four components: user model, emotions detection, interaction detection, and optimization. This approach considers the goals, preferences and knowledge of each individual user. The user's emotions are monitored and combined with the interaction. They then observe each part of the new approach individually and use, respectively, to create solutions to address each problem. After this, all parts are combined into a working prototype to evaluate the new approach.

Other studies introduce an approach for the design of an adaptive virtual training system based on the idea of universal design. "The system is based on a two-step approach that consists of an initial adaptation to the user capabilities and real-time adaptations during training based on measurements of the user. The adaptations concern the use of different representations of lessons with different complexity and interaction modalities. The proposed approach provides a flexible training system that can adapt to the needs of a broad group of users" (Loch et al., 2019)

In a framework developed by (Zahabi \& Abdul Razak, 2020) the authors take into account three fundamental components to adapt training system: (1) trainee's performance measurement; (2) adaptive variable; and (3) adaptive logic (Zahabi \& Abdul Razak, 2020). The first stage of this framework collects performance measures from a combination of online and real-time measurements, including information on the student's profile (experience, age, learning style), physiology (eye-tracking, heart rate, galvanic skin response), task performance (error and type), and kinematic/kinetic measures (range of motion, strength).

\section{Approach of immersive adaptive learning for VR simulator}

A simulator with adaptive learning must be capable of capturing the user's actions and reacting to them, being able to update and optimize the content. In this sense, the system must collect and classify all the information provided by the user, based on their emotions, behavior, skills and knowledge. Both correct and incorrect actions must be considered in the classification process. In this way, when the user performs incorrect actions, the system provides instant feedback and gives suggestions to the user. Feedback should be done in real-time, otherwise, it could lead to more errors, if would only be known at the end of the training. The system needs less optimization when the 
knowledge base about the user is enhanced. The approach proposed by the authors is structured of three main components: User Model (UM), Virtual Reality Scenario (VRS) and Adaptive Engine (AE) (Figure 1).

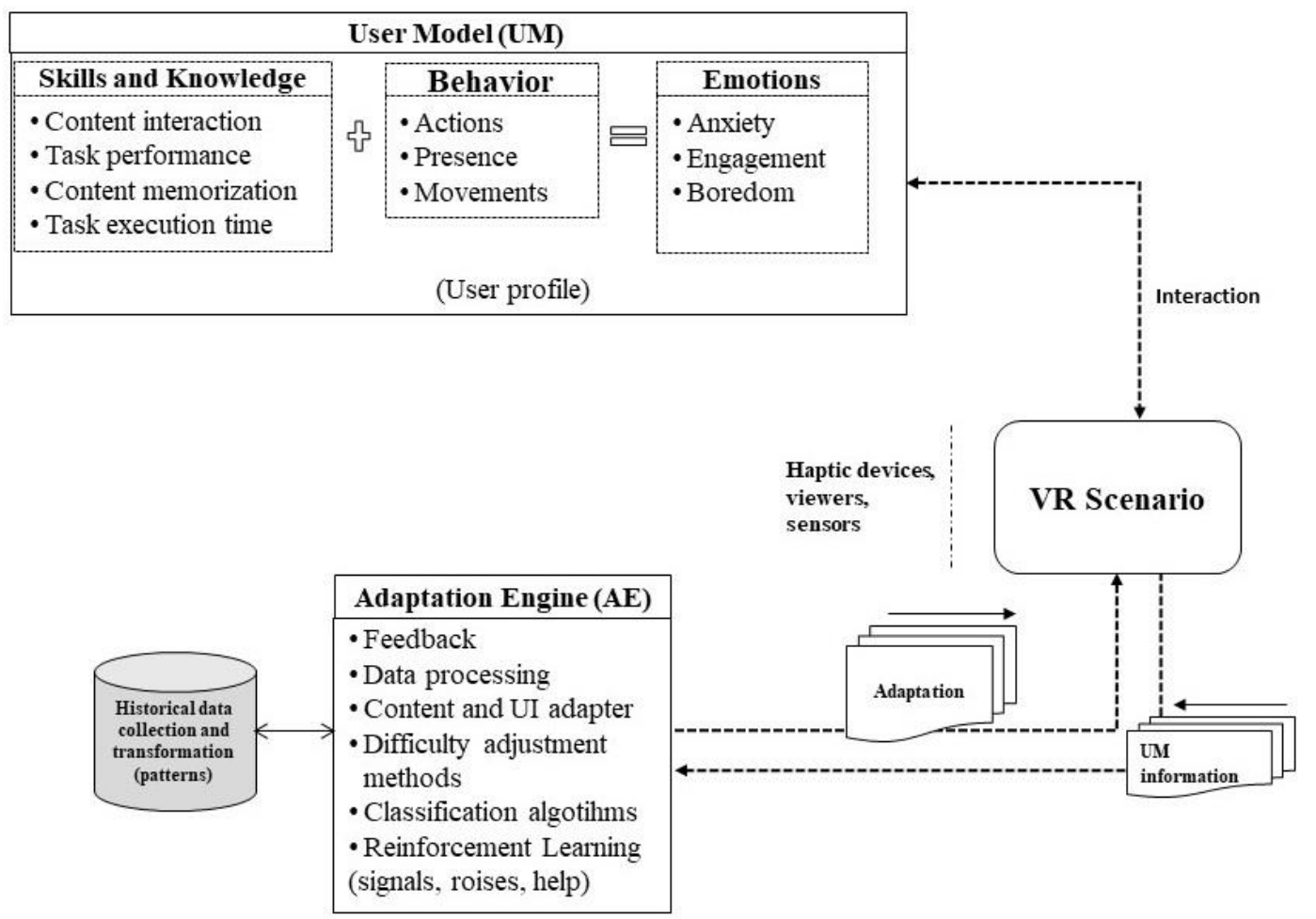

Figure 1. Summary of approach

$\mathrm{UM}$ is an essential component to represent the information about users to support the adaptation functionality of the systems (Brusilovsky \& Millán, 2007). Taking into account the proposed approach, the UM provides user characteristics splits into three main collections: Skills and Knowledge, Emotions, and Behavior. UM is in constant interaction with the other components and help to understand that the user has difficulties at some stage of training.

Skill and Knowledge: It provides a set of data related to user skills and knowledge. It groups together the performance of activities and the levels of knowledge acquired.

Emotions: It provides a set of data related to the user's emotions. These emotions are measured as variables in the VR scenario and are obtained through interaction devices with special emphasis on haptic devices. Some user emotions can be identified with the use devices such as pressure sensors, heart rate monitors among others. By supporting these accessories, it is possible to identify where they look or how they move to infer possible emotional states. A hypothesis to identify some emotions can start from the movements and/or the time of performance of an action. If a user remains a certain time without making movements or takes too long to perform an action, he could be confused, frustrated or bored. These emotions may have an impact on the user's skills and/or knowledge and may indicate whether the user has acquired a certain degree of knowledge about an activity or has achieved a skill. Some of the works consulted in this context refer to the Reinforcement Learning (RL) (Drugan, 2019; Li, Gomez, 
Nakamura, \& He, 2019; Rovira \& Slater, 2017; Sutton \& Barto, 2015). RL is a machine learning method involving an agent that can learn to perform a sequential decisionmaking task by interacting with an environment.

This approach mainly emphasizes three emotions-based variables: anxiety, engagement, and boredom. A dynamic adjustment of the difficulty is proposed to make from the AE to reach a balance between the challenge (activity) and the user's ability. Based on the flow theory (Csikszentmihalyi \& Csikzentmihaly, 1990) can be interpreted these emotional states.

Behavior: It provides a set of data related to user behavior. Behavioral data collection is closely related to emotions. Analyzing the user's behavior while performing an activity, it is possible to understand at what stage of learning they are to perform the activity. Therefore, it is possible to deduce what difficulty he presents during the training.

The VRS acts as a bridge with the AE, while the AE analyze the information provided by the VRS. The AE processes the information obtained and is supported by a collection of data containing behavior patterns for optimization and updating content and user interface (UI). During the adaptation process, the engine may lead to the process of $(\mathrm{RL})$ to generate efficient representations front of complex tasks. The idea is based on capturing the most important aspects of the real problem faced by the learning agent interacting with its environment to achieve a goal (Sutton \& Barto, 2015). Given a current state, the agent takes action and observes changes in the environment. RL agents have clear goals and can perceive aspects of their environment and choose actions to influence them.

The interaction of the components works as a cycle that improves the historical data collection. This collection is supported by haptic devices and other specialized technologies in the interpretation of emotions and behaviors. This collection promotes behavioral patterns and new transformations to AE. The adaptive engine classifies the content to display, and how to show it, in a way that helps the user to achieve an activity. This engine acquires the ability to reinforce the content, if necessary, and provide feedback to the other components. Following this idea, the approach will go through 3 stages: Configuration, Training, and Measurement.

Configuration: The input variables corresponding to the User Model are configured in this stage and prepares the environment for training. A simulator without previous information will need an initial configuration validated by experts that indicate the adequate average value of variables such as task execution time. For example, the appropriate average value of the task execution time variable in a surgery simulator should be validated by an expert surgeon, from the average time to perform a procedure in real life and the estimated average time to perform the same procedure in a simulator, with the intervention of controllers. Haptic gloves are preferably as controllers due to it provides more realism in the interaction compared to button controllers.

Training: The first training on a simulator starts from the initial configuration of the UM variables. This "calibration training" allows the user to become familiar with the environment and acquire skills with the controllers.

Measurement: This stage is the training result and involves a user classification algorithm, according to the skills acquired from data processing of information recorded during the training. A classification algorithm will place the user in one of the groups: 
beginner, intermediate, advanced. The user will move through these groups according to progress in the training. Placing the students in a group that represents their level of learning, this group could be considered for the transformation and adjustment of the content.

This operation cycle is closed at measurement and resumed at the configuration. Are compared the current UM records against the new training records from the first training of a user. The classification algorithm will determine whether the user stays in the same group or progresses to the next.

\section{Conclusions}

The authors' intention of this present study points out the main components of an approach of adaptive learning for a VR simulator. Future work will deep these components further to arrive at an adaptive learning model for VR simulators. The results of literature review revealed that the most common adaptive learning approaches conceive a user model to capture their actions and react to them through almost instantaneous feedback. The user characteristics must be considered to produce an appropriate adaptation to their learning progress during VR-based training. A VR simulator with adaptive learning must implement an adaptability engine to process information, adjust the content, and produce appropriate graphic changes in front of complex situations and tasks.

\section{References}

Brusilovsky, P., \& Millán, E. (2007). User models for adaptive hypermedia and adaptive educational systems. In The adaptive web (pp. 3-53). Springer.

Chen, S., \& Zhang, J. (2008). The Adaptive Learning System based on Learning Style and Cognitive State. Proceedings - 2008 International Symposium on Knowledge Acquisition and Modeling, KAM 2008. https://doi.org/10.1109/KAM.2008.60

Comas-González, Z., Echeverri-Ocampo, I., Zamora-Musa, R., Velez, J., Sarmiento, R., \& Orellana, M. (2017). Tendencias recientes de la educación virtual y su fuerte conexión con los entornos inmersivos. Espacios.

Csikszentmihalyi, M., \& Csikzentmihaly, M. (1990). Flow: The psychology of optimal experience (Vol. 1990). Harper \& Row New York.

De Troyer, O., Kleinermann, F., \& Ewais, A. (2010). Enhancing Virtual Reality Learning Environments with Adaptivity: Lessons Learned. https://doi.org/10.1007/978-3-642-16607-5_16

Drugan, M. M. (2019). Reinforcement learning versus evolutionary computation: A survey on hybrid algorithms. Swarm and Evolutionary Computation, 44, 228-246. https://doi.org/10.1016/j.swevo.2018.03.011

Kara, N., \& Sevim, N. (2020). Adaptive Learning Systems: Beyond Teaching Machines. Contemporary Educational Technology. https://doi.org/10.30935/cedtech/6095

Lele, A. (2013). Virtual reality and its military utility. Journal of Ambient Intelligence and Humanized Computing. https://doi.org/10.1007/s12652-011-0052-4

Lemke, C. (2013). Intelligent Adaptive Learning: An Essential Element of 21st Century 


\section{Teaching and Learning.}

Li, G., Gomez, R., Nakamura, K., \& He, B. (2019). Human-Centered Reinforcement Learning: A Survey. IEEE Transactions on Human-Machine Systems, 49(4), 337349. https://doi.org/10.1109/THMS.2019.2912447

Loch, F., Fahimipirehgalin, M., Czerniak, J. N., Mertens, A., Villani, V., Sabattini, L., ... Vogel-Heuser, B. (2019). An Adaptive Virtual Training System Based on Universal Design. IFAC-PapersOnLine, 51(34), 335-340. https://doi.org/10.1016/j.ifacol.2019.01.023

Paramythis, A., \& Loidl-Reisinger, S. (2004). Adaptive Learning Environments and eLearning Standards. Electronic Journal of E-Learning.

Pfandler, M., Lazarovici, M., Stefan, P., Wucherer, P., \& Weigl, M. (2017, September 1). Virtual reality-based simulators for spine surgery: a systematic review. Spine Journal, Vol. 17, pp. 1352-1363. https://doi.org/10.1016/j.spinee.2017.05.016

Real-Fernández, A., Molina-Carmona, R., Pertegal-Felices, M. L., \& Llorens-Largo, F. (2019). Definition of a feature vector to characterise learners in adaptive learning systems. Springer Proceedings in Complexity, 75-89. https://doi.org/10.1007/9783-030-30809-4_8

Rovira, A., \& Slater, M. (2017). Reinforcement Learning as a tool to make people move to a specific location in Immersive Virtual Reality. International Journal of Human Computer Studies, 98, 89-94. https://doi.org/10.1016/j.ijhcs.2016.10.007

Sutton, R. S., \& Barto, A. G. (2015). Reinforcement Learning: An Introduction Second edition, in progress.

Vaughan, N., Gabrys, B., \& Dubey, V. N. (2016, November 1). An overview of selfadaptive technologies within virtual reality training. Computer Science Review, Vol. 22, pp. 65-87. https://doi.org/10.1016/j.cosrev.2016.09.001

Weber, G. (2012). Adaptive Learning Systems. In Encyclopedia of the Sciences of Learning (pp. 113-115). https://doi.org/10.1007/978-1-4419-1428-6_534

Wojtok, A. J., Bab, S., \& Hirsch Martin. (2018, June). Adaptive learning in Virtual Reality: Current state and new approach. Retrieved from https://go-studyeurope.de/wp-content/uploads/2018/11/IRC2018-proceedings-final-web.pdf

Zahabi, M., \& Abdul Razak, A. M. (2020). Adaptive virtual reality-based training: a systematic literature review and framework. Virtual Reality, 1-28. https://doi.org/10.1007/s10055-020-00434-w 